\title{
Permanent genetic resources added to molecular ecology resources database 1 January 2009-30 April 2009.
}

\begin{abstract}
This article documents the addition of 283 microsatellite marker loci to the Molecular Ecology Resources Database. Loci were developed for the following species: Agalinis acuta; Ambrosia artemisiifolia; Berula erecta; Casuarius casuarius; Cercospora zeae-maydis; Chorthippus parallelus; Conyza canadensis; Cotesia sesamiae; Epinephelus acanthistius; Ficedula hypoleuca; Grindelia hirsutula; Guadua angustifolia; Leucadendron rubrum; Maritrema novaezealandensis; Meretrix meretrix; Nilaparvata lugens; Oxyeleotris marmoratus; Phoxinus neogaeus; Pristomyrmex punctatus; Pseudobagrus brevicorpus; Seiridium cardinale; Stenopsyche marmorata; Tetranychus evansi and Xerus inauris. These loci were cross-tested on the following species: Agalinis decemloba; Agalinis tenella; Agalinis obtusifolia; Agalinis setacea; Agalinis skinneriana; Cercospora zeina; Cercospora kikuchii; Cercospora sorghi; Mycosphaerella graminicola; Setosphaeria turcica; Magnaporthe oryzae; Cotesia flavipes; Cotesia marginiventris; Grindelia Xpaludosa; Grindelia chiloensis; Grindelia fastigiata; Grindelia lanceolata; Grindelia squarrosa; Leucadendron coniferum; Leucadendron salicifolium; Leucadendron tinctum; Leucadendron meridianum; Laodelphax striatellus; Sogatella furcifera; Phoxinus eos; Phoxinus rigidus; Phoxinus brevispinosus; Phoxinus bicolor; Tetranychus urticae; Tetranychus turkestani; Tetranychus ludeni; Tetranychus neocaledonicus; Tetranychus amicus; Amphitetranychus viennensis; Eotetranychus rubiphilus; Eotetranychus tiliarium; Oligonychus perseae; Panonychus citri; Bryobia rubrioculus; Schizonobia bundi; Petrobia harti; Xerus princeps; Spermophilus tridecemlineatus and Sciurus carolinensis.
\end{abstract}

Keyword: Molecular Ecology Resources Database; Loci; Microsatellite marker loci. 